\title{
Delay-Dependent Robust Passive Control for Uncertain Discrete-Time Systems with Time Delays ${ }^{*}$
}

\author{
Jufang Wang ${ }^{1}$, Changlong $\mathrm{Yu}^{1}$, Zhifeng Gao ${ }^{2}$ \\ ${ }^{1}$ College of Sciences, Hebei University of Science and Technology, Shijiazhuang, China \\ ${ }^{2}$ Nanjing University of Aeronautics and Astronautics, Nanjing, China \\ Email: wangjufang1981@126.com, changlongyu@126.com
}

Received April 19, 2013; revised May 20, 2013; accepted June 15, 2013

Copyright (c) 2013 Jufang Wang et al. This is an open access article distributed under the Creative Commons Attribution License, which permits unrestricted use, distribution, and reproduction in any medium, provided the original work is properly cited.

\begin{abstract}
This paper considers the problem of robust passive control for uncertain discrete systems with time-varying delays. We pay attention to designing a state feedback controller which guarantees the passivity of the closed-loop system for all admissible uncertainties. In terms of a linear matrix inequality, a sufficient condition for the solvability of this problem is presented and the explicit expression of the desired state feedback controller is given.
\end{abstract}

Keywords: Robust Passive Control; Discrete Systems; Time Delay

\section{Introduction}

In the past several years, much attention has been paid to the study of stability of systems with control input delay. Much of them is focused on the passivity analysis for classes of time-delay systems. Using classical definitions of passivity and positive realness, the conditions for a nonlinear system can be rendered passive via smooth state feedback, see [1,2]. The robust passive control problem for time-delay systems was dealt with in [3,4] via various approaches. The robust passivity synthesis problem for discrete-time-delay systems is investigated in $[5,6]$, but all these time delays are constant. To the best knowledge of authors, the problem of robust passive control for discrete-time systems with time-varying delays has not been fully investigated, which is more complex.

In this paper, we deal with the problem of robust passive feedback control for discrete systems with parameter uncertainties and time-varying delays. The parameter uncertainties are assumed to be time-varying but normbounded. The purpose is to construct a state feedback controller such that the closed-loop system is strictly passive and obtain a delay-dependent condition for the solvability of the problem.

*The project is supported by the Natural Science Foundation of China (10901045) and (11201112), and Natural Science Foundation of Hebei Province (A2013208147) and (A2011208012) and the Foundation of Hebei University of Science and Technology (XL200757).

\section{Statement of the Problem}

Consider the following uncertain discrete-time system with time-varying delays:

$$
\begin{aligned}
& x(k+1)=(A+\Delta A) x(k)+\left(A_{d}+\Delta A_{d}\right) x(k-\tau) \\
& +\left(D_{1}+\Delta D_{1}\right) w(k) \\
& z(k)=(E+\Delta E) x(k)+\left(E_{d}+\Delta E_{d}\right) x(k-\tau) \\
& +\left(D_{2}+\Delta D_{2}\right) w(k) \\
& x(k)=\phi(k), k \in[-\bar{\tau}, 0]
\end{aligned}
$$

where $x(k) \in R^{n}$ is the state, $z(k) \in R^{q}$ is the controlled output, $w(k) \in R^{p}$ the disturbance input which is assumed to belong to $l_{2}[0 ; \infty) ; \tau$ is a positive integer representing the time-varying delay of the system, which satisfies the following assumption: $0 \leq \tau \leq \bar{\tau}$. $\phi(k)$ is a real-valued initial function on $[-\bar{\tau}, 0]$; $A, A_{d}, D_{1}, E, E_{d}$ and $D_{2}$ are known real constant matrices; $\Delta A, \Delta A_{d}, \Delta D_{1}, \Delta E, \Delta E_{d}$ and $\Delta D_{2}$ are unknown matrices representing time-varying parameter uncertainties, and are assumed to be of the form

$$
\left[\begin{array}{ccc}
\Delta A & \Delta A_{d} & \Delta D_{1} \\
\Delta E & \Delta E_{d} & \Delta D_{2}
\end{array}\right]=\left[\begin{array}{l}
M_{1} \\
M_{2}
\end{array}\right] F(k)\left[\begin{array}{lll}
N_{1} & N_{2} & N_{3}
\end{array}\right]
$$

where $M_{1}, M_{2}, N_{1}, N_{2}$ and $N_{3}$, are known real constant matrices and $F(k)$ satisfies:

$$
F^{\mathrm{T}}(k) F(k)<I .
$$

Our problem is to establish the passive control for systems (2.1)-(2.3) to determine the conditions. To this end, we introduce the following fact and related definition of 
passivity.

Lemma 2.1 Given constant symmetric matrices $\Sigma_{1}$, $\Sigma_{2}, \quad \Sigma_{3}$, where $\Sigma_{1}=\Sigma_{1}^{\mathrm{T}}$, and $0<\Sigma_{2}=\Sigma_{2}^{\mathrm{T}}$, then $\Sigma_{1}+\Sigma_{3}^{\mathrm{T}} \Sigma_{2}^{-1} \Sigma_{3}<0$ if and only if

$$
\left[\begin{array}{cc}
\Sigma_{1} & \Sigma_{3}^{\mathrm{T}} \\
\Sigma_{3} & -\Sigma_{2}
\end{array}\right]<0 \text { or }\left[\begin{array}{cc}
-\Sigma_{2} & \Sigma_{3} \\
\Sigma_{3}^{T} & \Sigma_{1}
\end{array}\right]<0 \text {. }
$$

Lemma 2.2 Given constant matrices $\Sigma_{1}, \Sigma_{2}, \Sigma_{3}$ of appropriate dimensions with $\Sigma_{1}=\Sigma_{1}^{\mathrm{T}}$. Then

$$
\Sigma_{1}+\Sigma_{2} F(t) \Sigma_{3}+\Sigma_{3}^{\mathrm{T}} F^{\mathrm{T}}(t) \Sigma_{2}^{\mathrm{T}}<0 .
$$

where $F^{\mathrm{T}}(k) F(k)<I, \forall k$ if and only if for some scalar $\varepsilon>0$

$$
\Sigma_{1}+\varepsilon^{-1} \Sigma_{2} \Sigma_{2}^{\mathrm{T}}+\varepsilon \Sigma_{3}^{\mathrm{T}} \Sigma_{3}<0 .
$$

Lemma 2.3 Let $y(K)=x(k)-x(k-1)$, then the following inequality holds for any matrices $R, S_{1}$, $S_{2} \in R^{n \times n}$ and positive scalar $h>0$ :

$$
-\sum_{k-\tau}^{k-1} y^{\mathrm{T}}(i) R y(i) \leq \xi^{\mathrm{T}}(k)\left[\begin{array}{cc}
S_{1}^{\mathrm{T}}+S_{1} & -S_{1}^{\mathrm{T}}+S_{2} \\
* & -S_{2}^{\mathrm{T}}-S_{2}
\end{array}\right] \xi(k)+h \xi^{\mathrm{T}}(k)\left[\begin{array}{l}
S_{1}^{\mathrm{T}} \\
S_{2}^{\mathrm{T}}
\end{array}\right] R^{-1}\left[\begin{array}{ll}
S_{1}^{\mathrm{T}} & S_{2}^{\mathrm{T}}
\end{array}\right] \xi(k) .
$$

Definition 2.1 The dynamical systems (2.1) - (2.3) is called passive if there exists a scalar $\beta \geq 0$ such that

$$
\sum_{k=0}^{\infty} w^{\mathrm{T}}(k) z(k) \geq \beta, \forall \in L_{2}[0, \infty)
$$

where $\beta$ is some constant which depends on the initial condition of the system.

In addition, the systems (2.1)-(2.3) is said to be strictly passive if it is passive and $\left(D_{2}+\Delta D_{2}\right)>0$. In the sequel, we provide conditions under which a class of discrete- time linear dynamical systems with time-varying parameter uncertainties can be guaranteed to be strictly passive. First, we have the following result pertaining to the system (2.1)-(2.3).

\section{Proof of Main Results}

Theorem 3.1 The discrete-time systems with time delay (2.3) is strictly passive if there exist symmetric positive definite matrices $P, R, Q$ and $M_{1}, M_{2} \in R^{n \times m}$, such that the following LMI holds:

$$
\Xi=\left[\begin{array}{cc}
\Xi_{11} & P A_{d}-S_{1}^{\mathrm{T}}+S_{2}+\varepsilon N_{1}^{\mathrm{T}} N_{2} \\
* & -Q-S_{2}-S_{2}^{\mathrm{T}}+\varepsilon N_{2}^{\mathrm{T}} N_{2} \\
* & * \\
* & * \\
* & * \\
* & * \\
* & *
\end{array}\right.
$$

where $\Xi_{11}=P A+A^{\mathrm{T}} P+Q+S_{1}+S_{1}^{\mathrm{T}}+\varepsilon N_{1}^{\mathrm{T}} N_{1}$.

Proof. Choose a Lyapunov function candidate for the system (2.1) - (2.3) as follows:

$$
V(k)=x^{\mathrm{T}}(k) P x(k)+V_{1}(k)+V_{2}(k) .
$$

where

$$
\begin{aligned}
& V_{1}(k)=\sum_{\theta=-\tau+1}^{0} \sum_{i=k-1+\theta}^{k-1} y^{\mathrm{T}}(i) R y(i), \\
& V_{2}(k)=\sum_{i=k-\tau}^{k-1} x^{\mathrm{T}}(i) Q x(i), \\
& y(k)=x(k+1)-x(k) .
\end{aligned}
$$

Now, by some calculations, we can get that

$$
\left.\begin{array}{ccccc}
P D_{1}-E^{\mathrm{T}}+\varepsilon N_{1}^{\mathrm{T}} N_{3} & A^{\mathrm{T}} P & \tau A^{\mathrm{T}} R & \tau S_{1}^{\mathrm{T}} & P M_{1} \\
-E_{d}^{\mathrm{T}}+\varepsilon N_{2}^{\mathrm{T}} N_{3} & A_{d}^{\mathrm{T}} P & \tau A_{d}^{\mathrm{T}} R & \tau S_{2}^{\mathrm{T}} & 0 \\
-D_{2}-D_{2}^{\mathrm{T}}+\varepsilon N_{3}^{\mathrm{T}} N_{3} & D_{1}^{\mathrm{T}} P & \tau D_{1}^{\mathrm{T}} R & 0 & -M_{2} \\
* & -P & 0 & 0 & P M_{1} \\
* & * & -\tau R & 0 & \tau R M_{1} \\
* & * & * & -\tau R & 0
\end{array}\right]<0
$$

$$
\begin{aligned}
\Delta V(k)= & V(k+1)-V(k) \\
= & 2 x^{\mathrm{T}}(t) P y(k)+y^{\mathrm{T}}(k)(P+\tau R) y(k) \\
& -\sum_{i=k-\tau}^{k-1} y^{\mathrm{T}}(i) R y(i)+x^{\mathrm{T}}(t) Q x(t) \\
& -x^{\mathrm{T}}(t-\tau) Q x(t-\tau)
\end{aligned}
$$

We define that $\eta(k)=\operatorname{col}\{x(k), x(k-\tau), w(k)\}$, then have

$$
y(k)=\Gamma_{1} \eta(k) .
$$

From the Lemma 2.3, for $\forall S_{1}, S_{2} \in R^{n \times n}$, we can have that

$$
\begin{aligned}
-\sum_{k-\tau}^{k-1} y^{\mathrm{T}}(i) R y(i) \leq & {\left[\begin{array}{c}
x^{\mathrm{T}}(k) \\
x(k-\tau)
\end{array}\right]^{\mathrm{T}}\left[\begin{array}{cc}
S_{1}^{\mathrm{T}}+S_{1} & -S_{1}^{\mathrm{T}}+S_{2} \\
* & -S_{2}^{\mathrm{T}}-S_{2}
\end{array}\right]\left[\begin{array}{c}
x^{\mathrm{T}}(k) \\
x(k-\tau)
\end{array}\right] } \\
& +\tau\left[\begin{array}{c}
x^{\mathrm{T}}(k) \\
x(k-\tau)
\end{array}\right]\left[\begin{array}{l}
S_{1}^{\mathrm{T}} \\
S_{2}^{T}
\end{array}\right] R^{-1}\left[\begin{array}{ll}
S_{1}^{\mathrm{T}} & S_{2}^{\mathrm{T}}
\end{array}\right]\left[\begin{array}{c}
x^{\mathrm{T}}(k) \\
x(k-\tau)
\end{array}\right] .
\end{aligned}
$$


We have (3.3) and (3.4) into (3.2), after some manipulation, then obtain the following inequality:

$$
\begin{aligned}
& \Delta V(k)-2 z^{\mathrm{T}}(k) w(k) \\
& =V(k+1)-V(k)-2 z^{\mathrm{T}}(k) w(k) \\
& =\eta^{\mathrm{T}}(t)\left(\Theta+\Gamma_{1}^{\mathrm{T}} P \Gamma_{1}+\tau \Gamma_{1}^{\mathrm{T}} R \Gamma_{1}+\tau \Gamma_{2}^{\mathrm{T}} R^{-1} \Gamma_{2}\right) \eta(t) \\
& =\eta^{\mathrm{T}}(t) \tilde{\Theta} \eta(t)
\end{aligned}
$$

where

$\Theta$

$$
=\left[\begin{array}{ccc}
P A+A^{\mathrm{T}} P+Q+S_{1}+S_{1}^{\mathrm{T}} & P A_{d}-S_{1}^{\mathrm{T}}+S_{2} & P D_{1}-E^{\mathrm{T}} \\
* & -Q-S_{2}-S_{2}^{\mathrm{T}} & -E_{d}^{\mathrm{T}} \\
* & * & -D_{2}-D_{2}^{\mathrm{T}}
\end{array}\right]
$$

$\Gamma_{1}=\left[\begin{array}{lll}A & A_{d} & D_{1}\end{array}\right], \Gamma_{2}=\left[\begin{array}{lll}S_{1} & S_{2} & 0\end{array}\right]$.

If $\tilde{\Theta}<0$, then $\Delta V(k)-2 z^{\mathrm{T}}(k) w(k)<0$, and from which it follows that

$$
\begin{aligned}
\sum_{j=k_{0}}^{k_{f}} w^{\mathrm{T}}(j) z(j) & >\frac{1}{2} \sum_{j=k_{0}}^{k_{f}} \Delta V(k) \\
& =\frac{1}{2}\left[V\left(k_{0}\right)-V\left(k_{f}\right)\right]
\end{aligned}
$$

Since $V(k)>0$ for $x \neq 0$ and $V(k)=0$ for $x=0$, it follow as $k_{f} \rightarrow \infty$, that systems (2.1) - (2.3) is strictly passive. In view of Definition 2.1, the strictly passive condition is guaranteed if $\tilde{\Theta}<0$ and it can be expressed conveniently as

$$
\begin{aligned}
& {\left[\begin{array}{ccc}
P A+A^{\mathrm{T}} P+Q+S_{1}+S_{1}^{\mathrm{T}} & P A_{d}-S_{1}^{\mathrm{T}}+S_{2} & P D_{1}-E^{\mathrm{T}} \\
* & -Q-S_{2}-S_{2}^{\mathrm{T}} & -E_{d}^{\mathrm{T}} \\
* & * & -D_{2}-D_{2}^{\mathrm{T}}
\end{array}\right]} \\
& +\Gamma_{1}^{\mathrm{T}} P \Gamma_{1}+\tau \Gamma_{1}^{\mathrm{T}} R \Gamma_{1}+\tau \Gamma_{2}^{\mathrm{T}} R^{-1} \Gamma_{2}<0 .
\end{aligned}
$$

where $\Gamma_{1}=\left[\begin{array}{lll}A & A_{d} & D_{1}\end{array}\right], \Gamma_{2}=\left[\begin{array}{lll}S_{1} & S_{2} & 0\end{array}\right]$.

Application of Lemma 2.1 to the above inequality, it puts into the following form:

$\Pi=$

$$
\begin{aligned}
& {\left[\begin{array}{cccccc}
\Xi_{11} & P A_{d}-S_{1}^{\mathrm{T}}+S_{2} & P D_{1}-E^{\mathrm{T}} & A^{\mathrm{T}} P & \tau A^{\mathrm{T}} R & \tau S_{1}^{\mathrm{T}} \\
* & -Q-S_{2}-S_{2}^{\mathrm{T}} & -E_{d}^{\mathrm{T}} & A_{d}^{\mathrm{T}} P & \tau A_{d}^{\mathrm{T}} R & \tau S_{2}^{\mathrm{T}} \\
* & * & -D_{2}-D_{2}^{\mathrm{T}} & D_{1}^{\mathrm{T}} P & \tau D_{1}^{\mathrm{T}} R & 0 \\
* & * & * & -P & 0 & 0 \\
* & * & * & * & -\tau R & 0 \\
* & * & * & * & * & -\tau R
\end{array}\right]} \\
& <0 \\
& <0
\end{aligned}
$$

Substituting the uncertainty structure (2.5) into (3.9) and rearranging, we get the following inequality

$$
\Pi+\left[\begin{array}{c}
P M_{1} \\
0 \\
-M_{2} \\
P M_{1} \\
\tau R M_{1} \\
0
\end{array}\right] F(k)\left[\begin{array}{c}
N_{1}^{\mathrm{T}} \\
N_{2}^{\mathrm{T}} \\
N_{3}^{\mathrm{T}} \\
0 \\
0 \\
0
\end{array}\right]^{\mathrm{T}}\left[\begin{array}{c}
N_{1}^{\mathrm{T}} \\
N_{2}^{\mathrm{T}} \\
N_{3}^{\mathrm{T}} \\
0 \\
0 \\
0
\end{array}\right] F^{\mathrm{T}}(k)\left[\begin{array}{c}
M_{1}^{\mathrm{T}} P \\
0 \\
-M_{2}^{\mathrm{T}} \\
M_{1}^{\mathrm{T}} P \\
\tau M_{1}^{\mathrm{T}} R \\
0
\end{array}\right]^{\mathrm{T}}<0
$$

Then by Lemma 2.2, the inequality (3.10) holds if and only if for some $\varepsilon>0$

$$
\Pi+\varepsilon^{-1}\left[\begin{array}{c}
P M_{1} \\
0 \\
-M_{2} \\
P M_{1} \\
\tau R M_{1} \\
0
\end{array}\right]\left[\begin{array}{c}
M_{1}^{\mathrm{T}} P \\
0 \\
-M_{2}^{\mathrm{T}} \\
M_{1}^{\mathrm{T}} P \\
\tau M_{1}^{\mathrm{T}} R \\
0
\end{array}\right]+\varepsilon\left[\begin{array}{c}
N_{1}^{\mathrm{T}} \\
N_{2}^{\mathrm{T}} \\
N_{3}^{\mathrm{T}} \\
0 \\
0 \\
0
\end{array}\right]\left[\begin{array}{c}
N_{1}^{\mathrm{T}} \\
N_{2}^{\mathrm{T}} \\
N_{3}^{\mathrm{T}} \\
0 \\
0 \\
0
\end{array}\right]^{\mathrm{T}}<0
$$

for all admissible uncertainties satisfying (2.4). On using Lemma 2.1 in (3.11), it becomes that $\Xi<0$ in (3.1). This completes the proof.

\section{Robust Passive State Feedback Controller}

We now build on the foregoing results by considering the passive control problem, that is, designing a state feedback controller to render the closed-loop time-delay system passive. Extending the system (2.1)-(2.3), we consider a class of time-delay systems of the form:

$$
\begin{gathered}
x(k+1)=(A+\Delta A) x(k)+\left(A_{d}+\Delta A_{d}\right) x(k-\tau) \\
+\left(D_{1}+\Delta D_{1}\right) w(k)+\left(B_{1}+\Delta B_{1}\right) u(k) \\
z(k)=(E+\Delta E) x(k)+\left(E_{d}+\Delta E_{d}\right) x(k-\tau) \\
+\left(D_{2}+\Delta D_{2}\right) w(k)+\left(B_{2}+\Delta B_{2}\right) u(k)
\end{gathered}
$$

where $u(t) \in R^{n \times n}$ is the control input, $B_{1}, B_{2}$, are known real constant matrices; $\Delta B_{1}$ and $\Delta B_{2}$ are unknown matrices representing time-varying parametre uncertainties, and are assumed to be of the form:

$$
\left[\begin{array}{l}
\Delta B_{1} \\
\Delta B_{2}
\end{array}\right]=\left[\begin{array}{l}
M_{1} \\
M_{2}
\end{array}\right] F(k) N_{4} .
$$

Then the transformed system becomes

$$
\begin{aligned}
x(k+1) & =\left(A+\Delta A+B_{1} K+\Delta B_{1} K\right) x(k) \\
& +\left(A_{d}+\Delta A_{d}\right) x(k-\tau)+\left(D_{1}+\Delta D_{1}\right) w(k) \\
z(k)= & \left(E+\Delta E+B_{2} K+\Delta B_{2} K\right) x(k) \\
+ & \left(E_{d}+\Delta E_{d}\right) x(k-\tau)+\left(D_{2}+\Delta D_{2}\right) w(k)
\end{aligned}
$$

then we observe that 


$$
\left[\begin{array}{ccc}
\Delta A+\Delta B_{1} K & \Delta A_{d} & \Delta D_{1} \\
\Delta E+\Delta B_{2} K & \Delta E_{d} & \Delta D_{2}
\end{array}\right]=\left[\begin{array}{l}
M_{1} \\
M_{2}
\end{array}\right] F(k)\left[N_{1}+N_{4} K \quad N_{2} \quad N_{3}\right] .
$$

The following theorem establishes the main result.

Theorem 4.1 Consider the uncertain discrete-time delay system (4.4), (4.5). If there exists a positive scalar $\varepsilon>0$, a real matrix $Y$, three symmetric positive definite matrices $X, \tilde{Q}, R$ such that the following inequality holds:

$$
\left[\begin{array}{ccccccc}
\Phi_{11} & A_{d} X-W_{1}^{\mathrm{T}}+W_{2} & \Phi_{13} & \Phi_{14} & \Phi_{15} & \tau W_{1}^{\mathrm{T}} & X N_{1}^{\mathrm{T}}+Y^{\mathrm{T}} N_{4}^{\mathrm{T}} \\
* & -\tilde{Q}-W_{2}-W_{2}^{\mathrm{T}} & -X E_{d}^{\mathrm{T}} & X A_{d}^{\mathrm{T}} & \tau X A_{d}^{\mathrm{T}} & \tau W_{2}^{\mathrm{T}} & X N_{2}^{\mathrm{T}} \\
* & * & \Phi_{33} & D_{1}^{\mathrm{T}}-\varepsilon M_{2} M_{1}^{\mathrm{T}} & \Phi_{35} & 0 & X N_{3}^{\mathrm{T}} \\
* & * & * & -X+\varepsilon M_{1} M_{1}^{\mathrm{T}} & 0 & 0 & 0 \\
* & * & * & * & \Phi_{55} & 0 & 0 \\
* & * & * & * & * & -\tau X R X & 0 \\
* & * & * & * & * & * & -\varepsilon I
\end{array}\right]<0
$$

where

$$
\begin{aligned}
& \Phi_{11}=\left(A X+B_{1} Y\right)+\left(A X+B_{1} Y\right)^{\mathrm{T}}+\tilde{Q}+W_{1}+W_{1}^{\mathrm{T}}+\varepsilon M_{1} M_{1}^{\mathrm{T}}, \\
& \Phi_{13}=D_{1}-\left(E X+B_{2} Y\right)^{\mathrm{T}}-\varepsilon M_{1} M_{2}^{\mathrm{T}}, \\
& \Phi_{14}=\left(A X+B_{1} Y\right)^{\mathrm{T}}+\varepsilon M_{1} M_{1}^{\mathrm{T}}, \Phi_{15}=\tau\left(A X+B_{1} Y\right)^{\mathrm{T}}+\tau \varepsilon M_{1} M_{1}^{\mathrm{T}}, \\
& \Phi_{33}=-D_{2}-D_{2}^{\mathrm{T}}+\varepsilon M_{2} M_{2}^{\mathrm{T}}, \Phi_{35}=\tau D_{1}^{\mathrm{T}}-\tau \varepsilon M_{2} M_{1}^{\mathrm{T}}, \Phi_{55}=-\tau R^{-1}+\tau^{2} \varepsilon M_{1} M_{1}^{\mathrm{T}},
\end{aligned}
$$

then the systems (4.4), (4.5) are strictly passive, and the state-feedback gain matrix is given by $K=Y X^{-1}$.

Proof. Similar to Theorem 3.1.

Remark 4.1 It is noted that the matrix inequalities conditions in Theorem 4.1 are not LMIs. In order to solve the matrix inequalities conditions in Theorem 4.1, we can follow a similar line as in Lee et al. (2004) and Moon et al. (2001) to provide a nonlinear minimization problem subject to LMIs.

\section{REFERENCES}

[1] W. Lin, "Global Asymptotic Stabilization of General Nonlinear Systems with Stable Free Dynamics via Passivity and Bounded Feedback," Automatica, Vol. 32, No. 6, 1996, pp. 915-924. doi:10.1016/0005-1098(96)00013-1

[2] W. Z. Su and L. H. Xie, "Robust Control of Nonlinear Feedback Passive Systems," Automatica, Vol. 28, No. 2,
1996, pp. 5-93.

[3] B. T. Cui and M. G. Hua, "Robust Passive Control for Uncertain Discrete-Time Systems with Time-Varying Delays," Chaos, Solitons \& Fractals, Vol. 29, No. 2, 2006, pp. 331-341. doi:10.1016/j.chaos.2005.08.039

[4] Z. H. Guan, H. Zhang and S. H. Yang, "Robust Passive Control for Internet-Based Switching Systems with TimeDelay,” Chaos, Solitons \& Fractals, Vol. 36, No. 2, 2008, pp. 479-486. doi:10.1016/j.chaos.2006.06.039

[5] W. Lin and C. I. Byrnes, "Passivity and Absolute Stabilization of a Class of Discrete-Time Nonlinear Systems," Automatica, Vol. 31, No. 2, 1995, pp. 263-267. doi:10.1016/0005-1098(94)00075-T

[6] Q. K. Song, J. L. Liang and Z. D. Wang, "Passivity Analysis of Discrete-Time Stochastic Neural Networks with Time-Varying Delays," Neurocomputing, Vol. 72, No. 7-9, 2009, pp. 1782-1788. doi:10.1016/j.neucom.2008.05.006 